\title{
COMPORTAMENTO DA FUNÇÃO DO BANCO CENTRAL DO BRASIL: UMA ANÁLISE PARA O PERIODO DO SISTEMA DE METAS DE INFLAÇÃO
}

\author{
Richard Schnorrenberger' \\ Roberto Meurer $^{2}$
}

\section{Resumo}

A função de reação do Banco Central expressa as preferências da autoridade monetária em relação aos desvios da inflação à sua meta e ao hiato do produto. $\mathrm{O}$ presente trabalho investiga estas preferências e possíveis assimetrias nos objetivos do Banco Central do Brasil durante o período do sistema de metas para inflação, aplicando e estendendo o modelo de Clarida, Galí e Gertler (1999) com abordagem forward-looking. A econometria de séries temporais é empregada como principal ferramenta estatística para obtenção dos resultados. Os resultados indicam que o Banco Central do Brasil tem conduzido uma política monetária com preferências assimétricas em relação aos seus objetivos. O formulador de política monetária é mais sensível à estabilidade de preços, mas também se atenta aos movimentos do hiato do produto e suaviza intertemporalmente a taxa de juros de curto prazo. Ainda, a autoridade monetária reagiu com maior intensidade aos preços dos itens administrados nas gestões de Armínio Fraga e Henrique Meirelles e aos preços livres na gestão de Alexandre Tombini. Foram identificadas oscilações na função de reação em períodos de alta instabilidade financeira, quando ocorrem aumentos do peso da estabilidade de preços na decisão de política monetária. Por fim, o trabalho encontra indícios de ganhos de potência da política monetária ao longo do regime de metas, convergindo aos resultados de análises do Banco Central do Brasil.

Palavras-chave: função de reação, preferências, política monetária, Banco Central do Brasil, metas para a inflação

Classificação J.E.L.: E31, E32, E42, E52, E58

Graduado em Economia pela UFSC. Email: richard.schnr@gmail.com

Professor do Departamento de Economia da UFSC. Email: roberto.meurer@ufsc.br 


\section{INTRODUÇÃO}

A regra de política monetária propõe a utilização da taxa de juros de curto prazo como instrumento de política no objetivo de influenciar os desvios futuros do produto e da inflação em relação às suas metas. A regra, proposta primeiramente por Taylor (1993), sofreu alterações ao longo do tempo com o intuito de especificar o modelo mais apropriado para representar o comportamento da política monetária de diferentes países. A determinação da taxa de juros de curto prazo é derivada do problema de otimização intertemporal da autoridade monetária, que visa minimizar os custos sociais da inflação acima da meta e do emprego abaixo de seu nível potencial. Clarida, Galí e Gertler (1999) romperam com a abordagem backward-looking da regra de Taylor e introduziram as expectativas em relação à inflação e hiato do produto, estabelecendo uma função de reação forward-looking. Dessa forma, o Banco Central não estaria somente preocupado com os valores passados da inflação e do hiato do produto, mas também com seus valores futuros esperados.

Os fundamentos econômicos consideram que os policymakers podem apresentar preferências assimétricas em relação aos seus objetivos, isto é, o trade-off entre estabilidade de preços e desemprego é viesado. Em geral, os políticos e o público são comumente mais avessos a diferenças negativas do que a diferenças positivas do produto em relação ao produto potencial (CUKIERMAN, 2000). Semelhantemente, o alto custo social da inflação também torna os formuladores de política monetária mais avessos aos desvios positivos da inflação à meta. Aragón e Portugal (2009) mostram que a política monetária do Banco Central do Brasil (BCB) prioriza a estabilização da inflação durante a primeira década do regime de metas para inflação.

A experiência brasileira mostra ganhos de estabilidade macroeconômica no decorrer do regime de metas para inflação, no sentido de manter um nível de preços baixo e estável. Ao longo do regime de metas, a economia brasileira passou por alguns momentos de instabilidade financeira que podem ter provocado mudanças nas preferências da autoridade monetária. Da mesma forma, as alternâncias de gestão do Banco Central do Brasil podem ter causado ajustes assimétricos na função de reação. 
Neste trabalho, procura-se analisar o comportamento da política monetária e suas preferências ao longo do sistema de metas de inflação, de julho de 1999 ao final de 2013. Para isto, primeiramente é estimada a estrutura da economia de forma a verificar a dinâmica dos preços e mensurar a restrição da política monetária. Em seguida, são estimadas as funções de reação do Banco Central e os coeficientes recursivos, sendo aquelas modificadas conforme a análise requerida. As funções resposta da autoridade monetária são estimadas sob a ótica de uma economia forward-looking. Estas reações também são testadas com taxa de inflação separada em itens livres e administrados.

Além desta introdução, este trabalho é composto por 4 seções. Os modelos teóricos são apresentados na segunda seção. Na terceira seção, tem-se a especificação de estimação e a descrição das séries temporais. Na seção 4, são apresentados os resultados fundamentados nas estimativas da estrutura da economia e das funções de reação do BCB. Por fim, as conclusões do trabalho compõe a seção 5 .

\section{O MODELO TEÓRICO}

Com o objetivo de analisar assimetrias nas preferências do $\mathrm{BCB}$ ao longo do período de metas de inflação, emprega-se a base teórica e o modelo dinâmico de 3 equações Novo-Keynesiano proposto por Clarida, Gertler e Galí (1999) em relação à estrutura da economia. Este modelo permite que a autoridade monetária tenha preferências assimétricas em relação aos seus objetivos. A análise completa considera um sistema de 3 equações lineares com a inclusão de componentes de expectativas sobre as variações futuras de algumas variáveis. As duas primeiras equações teóricas dizem respeito à curva IS (equação 2.1) e curva de Phillips (equação 2.2), que expressam a dinâmica do produto em relação às variações da taxa real de juros e a a dinâmica da inflação, respectivamente:

$$
\begin{gathered}
y_{t}=-\varphi\left[r_{t}-E_{t} \pi_{t+1}\right]+E_{t} y_{t+1}+g_{t} \\
\pi_{t}=\lambda y_{t}+\beta E_{t} \pi_{t+1}+u_{t}
\end{gathered}
$$


onde $y_{t}$ representa o hiato do produto, $r_{t}$ é a taxa real de juros, $\pi_{t}$ representa a taxa de inflação, $E_{t} \pi_{t+1}$ representa a expectativa referente à taxa de inflação do período subsequente condicional às informações disponíveis em $t, E_{t} y_{t+1}$ representa a expectativa referente ao hiato do produto do período subsequente condicional às informações disponíveis em $t, g_{t}$ representa um choque de demanda e $u_{t}$ um choque de custos - ambos choques seguem um processo AR(1).

Através destas equações é possível calibrar os parâmetros da função de perda da autoridade monetária, terceira equação do modelo, e estabelecer a restrição que o policymaker enfrenta na otimização de seu problema. Por fim, a função perda descreve os objetivos da autoridade monetária e assim a possibilidade de analisar suas preferências intertemporais. O Banco Central utiliza a taxa nominal de juros de curto prazo para minimizar a variância do hiato do produto e inflação à sua meta. A função de reação remete à regra de Taylor com expectativas condicionais às informações disponíveis no presente, baseada em Clarida et al. (2000):

$$
i_{t}=\alpha+\beta\left(E_{t} \pi_{t+j}-\pi^{*}\right)+\delta\left(E_{t} y_{t+j}\right)
$$

onde, $i_{t}$ é a taxa nominal de juros a ser fixada pela autoridade monetária, $i^{*}$ é a taxa de juros de steady-state, $E_{t} \pi_{t+j}$ é a expectativa de inflação referente ao período futuro $j$ formada com o conjunto de informações disponíveis em $t$, $\pi^{*}$ é a meta de inflação anunciada pelo policymaker, e $E_{t} y_{t+j}$ é a expectativa de hiato do produto referente ao período futuro $j$ condicional ao conjunto de informações disponíveis em $t$. Os operadores de expectativas garantem que o formulador de política monetária age de maneira antecipada aos movimentos futuros dos preços e do nível de emprego.

Nossas estimativas mostrarão que o formulador da política monetária brasileira também incorpora e analisa as informações passadas, inclusive sobre a variação da taxa nominal de câmbio de forma indireta. Esse caráter da política monetária ocorre principalmente com o hiato do produto, que se mostrou mais significativo com defasagens passadas que através do operador de expectativas. Tanto Aragón e Portugal (2010), quanto Aragón e Medeiros (2011) e Minella et al. (2003) chegam à mesma evidência, na qual o Copom se mostra menos preocupado com o hiato do produto futuro 
quando fixa os níveis da taxa básica de juros. Desse modo, a regra de política monetária brasileira pode expressar uma visão forward-looking para o desvio da inflação à meta e backward-looking para o hiato do produto e depreciação da taxa nominal de câmbio $\left(\Delta q_{t}\right)$ :

$$
i_{t}=\alpha+\beta\left(E_{t} \pi_{t+j}-\pi^{*}\right)+\delta y_{t}
$$

A equação 2.4 é fundamental para investigar as preferências do formulador de política monetária. A estimação dos coeficientes $\beta$ e $\delta$ indicará se o Copom possui preferências assimétricas entre seus objetivos finais, isto é, se as decisões de política monetária revelam um viés de preferências. Por fim, a resolução do sistema se dá na análise conjunta das 3 equações apresentadas. $\mathrm{O}$ problema da política monetária consiste em escolher os níveis futuros desejados de produto e da taxa de inflação a fim de otimizar intertemporalmente a função de reação do Banco Central.

\section{METODOLOGIA E DADOS}

Para estimar as equações estruturais da economia brasileira e as funções de reação do BCB no período do Sistema de Metas de Inflação serão utilizados dados com observações trimestrais e mensais entre julho de 1999 e dezembro de 2013. Embora as amostras para o período do sistema de metas de inflação comecem em julho de 1999, a maioria das estimativas utilizam observações a partir de janeiro de 2000 , primeiro período em que há informações disponíveis no Relatório de Mercado Focus referente às expectativas dos agentes acerca da inflação futura. A desconsideração deste período de seis meses pode favorecer nossas estimações por representar um período de adaptação ao novo regime. As séries temporais foram obtidas no banco de dados do Instituto de Pesquisa Econômica Aplicada (IPEA), no Sistema Gerenciador de Séries Temporais (SGS) do BCB e no Sistema de Expectativas de Mercado do BCB que remetem ao Relatório de Mercado Focus ${ }^{3}$.

Disponível em https://www.bcb.gov.br/?FOCUSERIES?. O Relatório de Mercado Focus é um informativo semanal, divulgado pelo $\mathrm{BCB}$, que contém projeções feitas pelos analistas de mercado. Este trabalho utiliza a média das projeções feitas pelos analistas. Os dados de expectativas em relação ao IPCA estão disponíveis somente a partir de janeiro de 2000. 
O período escolhido para a análise é apropriado por englobar apenas regime de câmbio flutuante de metas de inflação. As séries com observações trimestrais serão utilizadas nas estimativas que compreendem a amostra integral por conterem menos ruído e os efeitos defasados da política monetária serem exibidos com menor número de defasagens. As séries com observações mensais serão utilizadas nas estimações que compreendem intervalos temporais menores, como estimações da função de reação dos diferentes mandatos presidenciais do Banco Central.

A estacionariedade das séries temporais será verificada para evitar a ocorrência de relação espúria entre as variáveis. O método dos Mínimos Quadrados Ordinários (MQO ou Ordinary Least Squares - OLS) será empregado nas estimativas da função de reação. Todos os modelos estimados serão submetidos aos testes de heterocedasticidade (teste $\mathrm{ARCH}$ ), autocorrelação serial (teste LM) e normalidade (teste Jarque-Bera). O software econométrico EViews 8 Student Version será utilizado na estimação das regressões e testes necessários.

A análise dos resíduos é composta pelos testes ARCH para verificar a hipótese de existência de heterocedasticidade nos resíduos; teste LM ou Breusch-Godfrey (BG) para verificar a autocorrelação nos resíduos; e teste Jarque-Bera para verificar a hipótese de normalidade dos resíduos estimados.

\subsection{Dados}

As séries temporais foram utilizadas com periodicidade mensal e trimestral, envolvendo o período entre janeiro de 2000 e dezembro de 2013:

i) Taxa Nominal de Juros $\left(i_{t}\right)$ : taxa de juros Selic ao ano definida pelo Copom. A transformação trimestral ocorreu por médias aritméticas simples da série mensal. Série 432 do SGS.

ii) Taxa de Inflação $\left(\pi_{t}\right)$ : Variação do IPCA acumulado nos últimos 12 meses. A transformação da série em trimestral ocorreu por média aritmética da série mensal. Série 433 do IBGE.

iii) Taxa Real de Juros $\left(r_{t}\right)$ : diferença entre a taxa nominal de juros $i_{t}$ e a taxa de inflação (IPCA). A transformação em trimestral foi por média aritmética da série mensal. 
iv) Hiato da Inflação $\left(\pi_{t}-\pi_{t}^{*}\right)$ : desvio da taxa de inflação em relação à meta vigente e definida pelo CMN, disponível em http://www.bcb.gov.br/pec/ relinf/normativos.asp. A transformação trimestral ocorreu por médias aritméticas simples da série mensal.

v) Hiato do Produto $\left(y_{t}\right)$ : diferença percentual entre o PIB com deflator implícito (Série do Ipeadata) e o PIB potencial. O PIB observado recebeu ajuste sazonal e o PIB potencial foi obtido através do filtro Hodrick-Prescott (HP). Desse modo, vale lembrar que o PIB potencial é estimado e não observado. A transformação trimestral ocorreu por médias aritméticas simples da série mensal.

vi) Preços Administrados $\left(\mathrm{Pa}_{t}\right)$ : Variação do índice de preços (IPCA) acumulado nos últimos 12 meses que engloba itens administrados por contrato e monitorados como o IPTU, taxa de água e esgoto, gás de bujão, gás encanado, energia elétrica residencial e transporte coletivo (ônibus). A transformação trimestral ocorreu por médias aritméticas simples da série mensal. Série 4449 do SGS.

vii) reços Livres $\left(\mathrm{Pl}_{t}\right)$ : Variação do índice de preços (IPCA) acumulado nos últimos 12 meses que exclui os preços monitorados ou administrados. A transformação trimestral ocorreu por médias aritméticas simples da série mensal. Série 11428 do SGS.

viii) Taxa Nominal de Câmbio $\left(q_{t}\right)$ : definida como $\ln \left(q_{t}\right)$, onde $q_{t}$ é a média mensal da taxa nominal de câmbio diária - Série 3696 do SGS. A depreciação da taxa nominal de câmbio é representada por $\Delta q_{t}$. A transformação trimestral ocorreu por médias aritméticas simples da série mensal.

A introdução de operadores de expectativas nas regras de Taylor com abordagem forward-looking demanda a criação de novas variáveis que levem em consideração a estrutura e dinâmica da economia brasileira. Como o Brasil utiliza um sistema de metas para a inflação no final de períodos anuais, o operador de expectativas deve incorporar as expectativas para o ano vigente e o próximo de maneira ponderada. A sugestão surge do trabalho de Minella et al. (2002), com as expectativas referentes aos desvios futuros da inflação em relação à meta tomando a seguinte forma: 


$$
E_{\pi, j}=\left(\frac{12-j}{12}\right)\left(E_{j} \pi_{t}-\pi_{t}^{*}\right)+\left(\frac{j}{12}\right)\left(E_{j} \pi_{t+1}-\pi_{t+1}^{*}\right)
$$

onde $j$ é a referência do mês em que as expectativas foram formadas. As expectativas utilizadas nas séries ponderadas são referentes às previsões de mercado (Relatório de Mercado Focus) do primeiro dia útil de cada mês. A ponderação dessas variáveisreflete a influência da época do ano na formação das expectativas, isto é, quanto mais próximo ao final do ano mais preocupado o BCB está com a inflação esperada do ano seguinte. Do contrário, períodos anuais entre o primeiro e segundo trimestre expressam maiores preocupações com o nível das variáveis no ano vigente. As variáveis abaixo seguem a estrutura do desvio ponderado da inflação esperada em relação à meta de inflação $\left(E_{\pi, j}\right)$ da equação 3.1 e agem como operadores ponderados de expectativas:

i) Desvio ponderado do produto esperado em relação ao produto potencial $\left(E_{y, j}\right)$ :

$E_{y, j}=\left(\frac{12-j}{12}\right)\left(E_{j} y_{t}\right)+\left(\frac{j}{12}\right)\left(E_{j} y_{t+1}\right)$, onde $j$ é a referência do mês em que as expectativas foram formadas.

ii) Desvio ponderado da inflação esperada de preços administrados $\left(E_{P a, j}\right)$ :

$E_{P a, j}=\left(\frac{12-j}{12}\right)\left(E_{j} P a_{t}\right)+\left(\frac{j}{12}\right)\left(E_{j} P a_{t+1}\right)$, onde $j$ é a referência do mês em que as expectativas foram formadas.

iii) Desvio ponderado da inflação esperada de preços livres $\left(E_{P l, j}\right)$ :

$E_{P l, j}=\left(\frac{12-j}{12}\right)\left(E_{j} P l_{t}\right)+\left(\frac{j}{12}\right)\left(E_{j} P l_{t+1}\right)$, onde $j$ é a referência do mês em que as expectativas foram formadas.

Assim como Aragón e Portugal (2009), algumas estimativas utilizam variáveis binárias dummies para captar choques macroeconômicos e prevenir o problema de heterocedasticidade. A variável dummy $D_{\pi, 2002}$ (=1 para 2002T4, e 0 caso contrário) captura o ciclo de inflação alta iniciado no último trimestre de 2002, compreendendo o período de forte instabilidade política e depreciação acentuada do real. A variável dummy $D_{y, 2003}$ (=1 para 2003T1-2003T2, e 0 caso contrário) captura a retração 
intensa da atividade interna advinda de condições adversas do crédito, persistência de incertezas macroeconômicas e recrudescimento da inflação. A variável dummy $D_{y, 2009}$ (=1 para 2008M12-2009M07, e 0 caso contrário) é utilizada nas estimativas da função de reação específica da gestão de Henrique Meirelles na presidência do Banco Central. $D_{y, 2009}$ captura os efeitos da crise do subprime de 2008 que causou forte queda nas expectativas de produto, disseminando impactos negativos sobre a atividade econômica de 2009.

\section{RESULTADOS E DISCUSSÃO}

\subsection{Testes de Estacionariedade}

Antes de estimar os modelos teóricos descritos anteriormente, foram testadas as condições de estacionariedade das variáveis através dos testes ADF (Augmented Dickey-Fuller), Phillips-Perron (PP) e Kwiatkowski-Phillips-Schmidt-Shin (KPSS). Para os testes ADF e PP a hipótese nula é que a série temporal apresenta raíz unitária. Por outro lado, o teste KPSS testa a hipótese nula de que a série é estacionária. No teste ADF, o critério de informação de Schwarz foi utilizado para escolher o número ótimo de defasagens considerando um número máximo de defasagens iguais a 10. Nos testes PP e KPSS, o estimador automático Newey-West e a função Bartlett Kernel foram utilizadas para determinar a banda ótima de defasagens. Ainda, houve a inclusão de componentes determinísticos - constante (c) e tendência linear (t) - nos testes quando os mesmos foram estatisticamente significativos.

Tabela 1: Resultados dos testes de raíz unitária para as séries trimestrais

\begin{tabular}{|c|c|c|c|c|c|}
\hline Variáveis & $\begin{array}{c}\text { Regressores } \\
\text { Exógenos }\end{array}$ & Teste ADF & Teste PP & Teste KPSS & $\begin{array}{c}\text { Série } \\
\text { Estacionária }\end{array}$ \\
\hline$i_{t}$ & $\mathrm{c}, \mathrm{t}$ & $-3,0828^{\mathrm{ns}}$ & $-2,6928^{\mathrm{ns}}$ & $0,1002^{\mathrm{ns}}$ & Não conclusivo \\
\hline$i_{t}$ & $\mathrm{c}$ & - & - & $0,8632^{* * * *}$ & Sim \\
\hline$\Delta i_{t}$ & - & $-6,3423^{* * *}$ & $-3,0019^{* * *}$ & $0,0620^{\mathrm{ns}}$ & Não conclusivo \\
\hline$r_{t}$ & $\mathrm{c}, \mathrm{t}$ & $-2,7748^{\mathrm{ns}}$ & $-2,6033^{\text {ns }}$ & $0,1522^{* *}$ & Não \\
\hline$\Delta r_{t}$ & - & $-5,7425^{* * * *}$ & $-6,2863^{* * *}$ & $0,0654^{\mathrm{ns}}$ & Sim \\
\hline$\pi_{t}$ & $\mathrm{c}$ & $-2,5539^{*}$ & $-2,3842^{\mathrm{ns}}$ & $0,3518^{*}$ & Não conclusivo \\
\hline$\Delta \pi_{t}$ & - & $-3,2730^{* * *}$ & $-3,7109^{* * *}$ & $0,0423^{\mathrm{ns}}$ & Sim \\
\hline
\end{tabular}




\begin{tabular}{c|c|l|l|l|c}
\hline$\pi_{t}-\pi_{t}^{*}$ & $\mathrm{c}, \mathrm{t}$ & $-3,9674^{* *}$ & - & $0,0933^{\mathrm{ns}}$ & $\operatorname{Sim}$ \\
\hline$\pi_{t}-\pi_{t}^{*}$ & - & $-2,0829^{* *}$ & $-1,8528^{*}$ & $0,3024^{\mathrm{ns}}$ & $\operatorname{Sim}$ \\
\hline$E_{\pi, j}$ & - & $-1,6190^{*}$ & $-1,7415^{*}$ & $0,1924^{\mathrm{ns}}$ & Sim \\
\hline$y_{t}$ & - & $-4,4158^{* * *}$ & $-2,6905^{* * *}$ & $0,0868^{\mathrm{ns}}$ & Sim \\
\hline$E_{y, j}$ & $\mathrm{c}, \mathrm{t}$ & $-3,4816^{*}$ & - & $0,1115^{\mathrm{ns}}$ & Sim \\
\hline$E_{y, j}$ & - & $-2,9296^{* * *}$ & $-1,8053^{*}$ & $0,4125^{*}$ & Não conclusivo \\
\hline$P l_{t}$ & $\mathrm{c}$ & $-2,7489^{*}$ & $-2,1547^{\mathrm{ns}}$ & $0,0784^{\mathrm{ns}}$ & Não conclusivo \\
\hline$P a_{t}$ & $\mathrm{c}, \mathrm{t}$ & $-2,2432^{\mathrm{ns}}$ & $-2,9534^{\mathrm{ns}}$ & $0,1043^{\mathrm{ns}}$ & Não conclusivo \\
\hline$\Delta P a_{t}$ & - & $-4,3471^{* * *}$ & $-6,6702^{* * *}$ & $0,0631^{\mathrm{ns}}$ & Sim \\
\hline$E_{P a, j}$ & $\mathrm{c}$ & $-2,1518^{\mathrm{ns}}$ & $-4,5479^{* * *}$ & $0,5494^{* *}$ & Não conclusivo \\
\hline$\Delta E_{P a, j}$ & - & $-4,9983^{* * *}$ & $-5,014^{* * *}$ & $0,6596^{* *}$ & Não conclusivo \\
\hline$q_{t}$ & $\mathrm{c}, \mathrm{t}$ & $-2,8563^{\mathrm{ns}}$ & $-2,2061^{\mathrm{ns}}$ & $0,1198^{*}$ & Não \\
\hline$\Delta q_{t}$ & - & $-5,772^{* * *}$ & $-4,8169^{* * *}$ & $0,1763^{\mathrm{ns}}$ & Sim \\
\hline
\end{tabular}

Nota: ${ }^{*}$ Significativo a $10 \%,{ }^{* *}$ Significativo a $5 \%,{ }^{* * *}$ Significativo a $1 \%,{ }^{\text {ns }}$ Não-significativo,

Fonte: Elaboração própria/Eviews

Conforme as estimativas para verificação de estacionariedade das séries temporais, exibidas na tabela 1 , somente as variáveis taxa real de juros e taxa nominal de câmbio são estacionárias unicamente em primeira diferença, considerando um nível mínimo de significância de 10\%. As outras séries, como a taxa nominal de juros, taxa de inflação, hiato da inflação e sua expectativa, hiato do produto e sua expectativa, preços livres, preços administrados e sua expectativa, mostraram ser estacionárias em nível. Nos casos em que houve divergências entre os testes para a variável em nível também foi efetuado o teste para a variável em primeira diferença. Devido às divergências, estas variáveis (taxa nominal de juros, taxa de inflação e taxa de inflação esperada de preços administrados) foram utilizadas tanto em nível quanto em primeira diferença na estimação dos modelos.

Semelhantemente, os testes de raíz unitária para as séries mensais, que serão utilizadas nas estimativas com intervalos temporais mais curtos, apresentaram resultados convergentes aos das séries trimestrais, com exceção da inflação dos preços administrados, que agora passou a ser estacionária em nível.

\subsection{Estimativas dos Modelos Teóricos: Análises para o Período Todo}

A curva IS e a curva de Phillips a serem estimadas seguem as equações teóricas 4.1 e 4.2 respectivamente: 


$$
\begin{aligned}
& y_{t}=\alpha_{0}+\alpha_{1} y_{t-1}+\alpha_{2} y_{t-2}+\alpha_{3} y_{t-4}+\alpha_{4} \Delta r_{t-2} \\
& \pi_{t}=\alpha_{1} \pi_{t-1}+\alpha_{2} \pi_{t-2}+\alpha_{3} \pi_{t-5}+\alpha_{4} E_{\pi, j}+\alpha_{5} y_{t}+\alpha_{6} \Delta q_{t-1}
\end{aligned}
$$

As estimativas são apresentadas pelas tabelas 2 e 3. Em geral, os testes de especificação garantem resíduos estimados bem comportados já que nenhuma das hipóteses nulas dos testes ARCH, LM ou Jarque-Bera foi rejeitada. Grande parte dos coeficientes de ambas equações estimadas são significativos e demonstram uma convergência aos sinais esperados. As defasagens dos modelos foram selecionadas através do critério de defasagem ótima de Akaike, Schwarz e Hannan-Quinn. Seguindo o método geral-específico (HENDRY, 2001), na maior parte dos modelos estimados foram excluídas as variáveis não significativas ao nível de 10\%.

Tabela 2: Estimativas da Curva IS

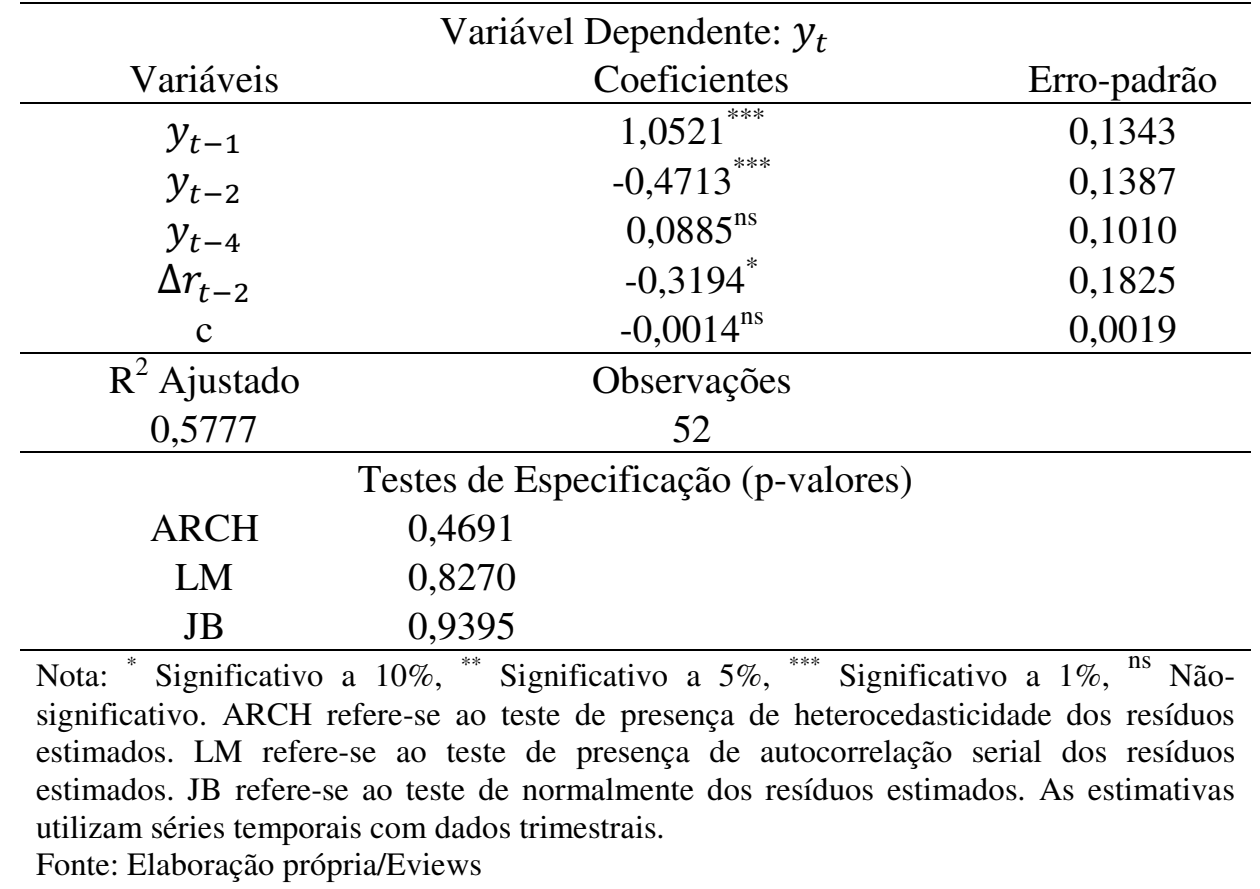


Tabela 3: Estimativas da Curva de Phillips

\begin{tabular}{ccc}
\hline & $\begin{array}{c}\text { Variável Dependente: } \pi_{t} \\
\text { Coeficientes }\end{array}$ & Erro-padrão \\
\hline$\pi_{t-1}$ & $1,2369^{* * * *}$ & 0,1112 \\
$\pi_{t-2}$ & $-0,4892^{* * *}$ & 0,1052 \\
$\pi_{t-5}$ & $0,1546^{* * *}$ & 0,0344 \\
$E_{\pi, j}$ & $0,5562^{* * *}$ & 0,1245 \\
$y_{t}$ & $0,1354^{* *}$ & 0,0522 \\
$\Delta q_{t-1}$ & $0,0268^{*}$ & 0,0149 \\
\hline $\mathrm{R}^{2}$ Ajustado & Observações \\
0,9339 & 51 & \\
\hline
\end{tabular}

Testes de Especificação (p-valores)

$\mathrm{ARCH}$

0,6256

LM

0,2063

JB

0,2955

Nota: ${ }^{*}$ Significativo a $10 \%,{ }^{* *}$ Significativo a $5 \%,{ }^{* * *}$ Significativo a $1 \%,{ }^{\text {ns }}$ Nãosignificativo. ARCH refere-se ao teste de presença de heterocedasticidade dos resíduos estimados. LM refere-se ao teste de presença de autocorrelação serial dos resíduos estimados. JB refere-se ao teste de normalmente dos resíduos estimados. As estimativas utilizam séries temporais com dados trimestrais.

Fonte: Elaboração própria/Eviews

Na curva IS, o coeficiente que mede o impacto da taxa real de juros no hiato do produto foi estatisticamente significativo a $10 \%$, revelando que uma redução de $1 \%$ na primeira diferença da taxa real de juros gera um aumento de aproximadamente 0,32 pontos percentuais no hiato do produto de dois trimestres à frente. Desse modo, confirma-se a teórica relação inversa entre o produto e a taxa real de juros. Quanto à curva de Phillips, a taxa de inflação presente mostrou "carregar" efeitos do passado e antecipar os movimentos da inflação futura esperada pelo mercado; cerca de 55\% da inflação esperada é incorporada nos preços presentes. $\mathrm{O}$ hiato do produto corrente e a depreciação da taxa de câmbio defasada em um trimestre também mostraram impactar significativamente a taxa de inflação. Conforme as estimativas, o efeito pass-through da taxa de câmbio sobre o nível de preços é pequeno. Porém, o mesmo pode estar incorporado no operador de expectativas de inflação $E_{\pi, j}$, agindo de forma indireta sobre a inflação. Ainda, conforme Tostes (2014) o aumento da credibilidade do Banco Central e da política 
fiscal ao longo do regime de metas contribui para reduzir o pass-through do câmbio sobre a inflação de preços livres ao longo do tempo.

Diferentemente do câmbio, o hiato do produto consegue gerar um efeito direto de longo prazo de aproximadamente 0,26 pontos percentuais sobre a taxa de inflação. Isso acontece por meio dos componentes autorregressivos que amplificam o seu efeito. Os componentes autorregressivos da curva de Phillips estimada produzem o efeito de longo prazo. Um aumento de $1 \%$ do hiato do produto em $t$ gera uma inflação de 0,13 pontos percentuais em $t$, mais $0,13 \% \mathrm{x} 1,23$ em $t+1$, mais $0,13 \% \mathrm{x}-0,48$ em $t+2$, mais $0,13 \% \mathrm{x} 0,15$ em $t+5$. A variável explicativa $\pi_{t-5}$ revela que o efeito de longo prazo leva 5 trimestres para se esgotar completamente. Assim, os efeitos do hiato do produto sobre os preços estão sendo incorporados pelas expectativas de mercado em relação à inflação futura.

Analisando a estrutura da economia, a taxa real de juros impacta indiretamente nos preços levando dois trimestres para operar completamente. Isto ocorre porque o impacto final de 0,32 pontos percentuais da taxa real de juros sobre o hiato do produto em $t+2$ será levado à inflação através do elemento $y_{t}$ da curva de Phillips. Logo, esta variação inicial da taxa real de juros causa um efeito multiplicativo de 0,04 pontos percentuais na inflação de $t+2$. Este advém da multiplicação dos $0,32 \%$ pelo impacto de 0,13 pontos percentuais de $y_{t+2}$ sobre $\pi_{t+2}$.

Uma vez analisada a estrutura da economia e definidas as restrições para a política monetária, é possível estimar a função de reação linear para analisar as preferências do BCB. A equação abaixo representa a função de reação a ser estimada:

$i_{t}=\alpha_{1} i_{t-1}+\alpha_{2} i_{t-2}+\alpha_{3} i_{t-3}+\alpha_{4} E_{\pi, j}+\alpha_{5} y_{t-1}+\alpha_{6} D_{y, 2003}$

A tabela 4 exibe as estimativas da função de reação linear que abrange o período completo do regime de metas para inflação. Em geral, os coeficientes estimados da equação 4.3 são estatisticamente diferentes de zero e apresentam convergência aos fundamentos da regra de Taylor. Os testes de especificação apontam resíduos ruído branco para o modelo estimado. As decisões do Copom revelam uma maior preocupação com os desvios da inflação futura à sua meta, ou seja, o impacto da variável $E_{\pi, j}$ sobre a taxa 
de juros corrente é maior do que o impacto de $y_{t-1}$. Conforme as estimativas, um aumento de $1 \%$ no desvio ponderado da inflação esperada em relação à meta de inflação provoca um incremento de 0,58 pontos percentuais na Selic, enquanto que um aumento de $1 \%$ no hiato do produto em $t-1$ gera um efeito positivo de apenas 0,15 pontos percentuais na Selic. A variável $\Delta q_{t}$ apresentou-se estatisticamente não significativa como variável explicativa. Conforme as estimativas da curva de Phillips, esse fato pode indicar que os efeitos do mercado cambial já estão incorporados nas expectativas futuras de inflação. A variável dummy $D_{y, 2003}$ indica que a queda expressiva na atividade econômica dos primeiros trimestres de 2003 interferiu nas decisões de política monetária, que foi relaxada a partir do terceiro trimestre daquele ano. Por fim, o nível do $\mathrm{R}^{2}$ ajustado revela que o modelo estimado captou cerca de $97 \%$ das informações que influenciam na determinação da taxa Selic, demonstrando alto poder de explicação da função de reação estimada.

Tabela 4: Estimativas da função de reação com taxa Selic em nível

\begin{tabular}{ccc}
\hline \multicolumn{3}{c}{ Variável Dependente: $i_{t}$} \\
Variáveis & $1,6636^{* * * *}$ & Erro-padrão \\
\hline$i_{t-1}$ & $-1,2632^{* * *}$ & 0,1331 \\
$i_{t-2}$ & $0,5593^{* * *}$ & 0,1998 \\
$i_{t-3}$ & $0,5827^{* * *}$ & 0,0989 \\
$E_{\pi, j}$ & $0,1545^{* *}$ & 0,1524 \\
$y_{t-1}$ & $-0,0227^{* *}$ & 0,0620 \\
$D_{y, 2003}$ & Observações \\
\hline $\mathrm{R}^{2}$ Ajustado & 53 & 0,0104 \\
0,9677 & \multicolumn{3}{c}{} \\
\hline \multicolumn{4}{c}{ Testes de Especificação (p-valores) } \\
LM & 0,7624 & \\
JB & 0,7318 & \\
\hline
\end{tabular}

Nota: ${ }^{*}$ Significativo a $10 \%,{ }^{* *}$ Significativo a $5 \%,{ }^{* * *}$ Significativo a $1 \%,{ }^{\text {ns }}$ Nãosignificativo. ARCH refere-se ao teste de presença de heterocedasticidade dos resíduos estimados. LM refere-se ao teste de presença de autocorrelação serial dos resíduos estimados. JB refere-se ao teste de normalmente dos resíduos estimados. As estimativas utilizam séries temporais com dados trimestrais.

Fonte: Elaboração própria/Eviews 
Os primeiros parâmetros da equação estimada mostram que a taxa nominal de juros é suavizada ao longo do tempo, isto é, a política monetária é ajustada gradualmente através de ciclos de aperto ou relaxamento monetário. Aragón e Portugal (2009) citam algumas razões para que os policymakers não realizem variações excepcionais do instrumento de política monetária:

i) pequenas e persistentes variações da taxa de juros de curto prazo geram um efeito maior de transmissão da política monetária;

ii) quando necessário, é mais fácil corrigir e reverter a política monetária;

iii) grandes flutuações (volatilidade) da taxa nominal de juros podem desestabilizar os mercados financeiros e cambiais.

Para complementar a análise de preferências da política monetária brasileira, estima-se os coeficientes recursivos da função de reação estimada. Esta ferramenta permite analisar a evolução do coeficiente ao longo do período através da adição de observações a cada novo intervalo. A cada intervalo progressivo de tempo é realizada uma nova análise de regressão entre as variáveis, com o intuito de analisar o peso de cada um dos componentes ao longo do tempo. As figuras 1 e 2 apresentam esta análise para as variáveis objetivo da política monetária, onde o primeiro intervalo compreende o período 2000T1-2003T2.

Figura 1: Coeficiente recursivo da variável

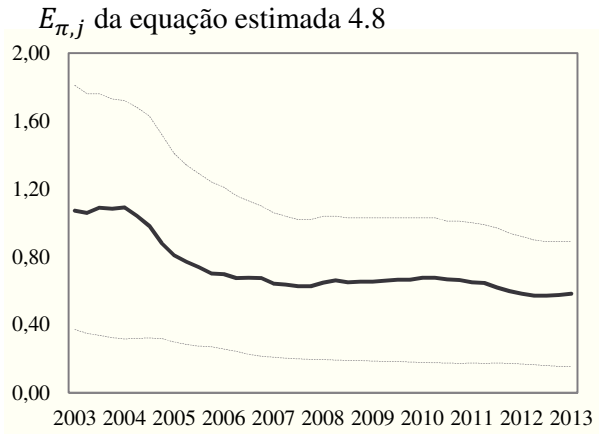

Fonte: Elaborado pelo autor/Eviews
Figura 2: Coeficiente recursivo da variável

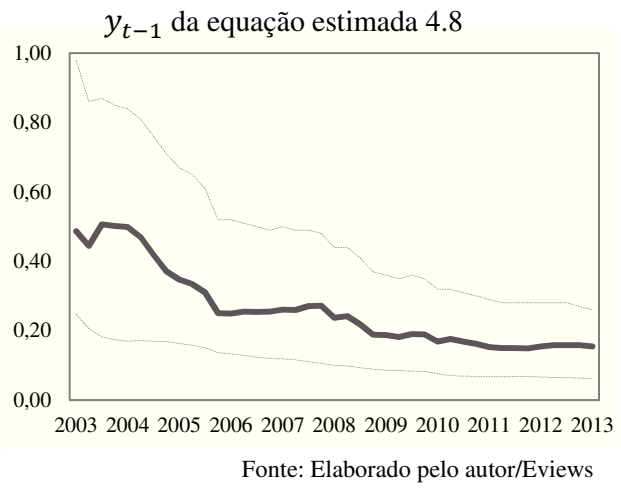


O gráfico evidencia claramente que há uma assimetria de preferências na função de reação do $\mathrm{BCB}$, com maior peso ao controle de preços, e que as duas variáveis objetivo da política monetária possuem trajetórias descendentes dos seus respectivos coeficientes recursivos. Mais importante, tanto o desvio ponderado da inflação esperada quanto o hiato do produto defasado em 1 trimestre diminuem seus impactos e influências sobre as decisões do Copom. Há dois motivos razoáveis para esta evidência:

i) Ganhos de potência da política monetária, isto é, o Banco Central precisa executar uma política monetária menos intensiva para gerar as respostas desejadas no produto e na taxa de inflação. Conforme Relatório de Inflação de março de 2012, a potência da política monetária no Brasil tem aumentado nos últimos anos. Isto também é explicitado no boxe "Poder da Política Monetária no Brasil", publicado no Relatório de Inflação de junho de 2010. Essa maior potência da política monetária remete ao amadurecimento das instituições, o aprofundamento do mercado de crédito e alongamento dos contratos;

ii) O tamanho do afastamento da taxa de inflação em relação à meta e do hiato do produto pode influenciar na intensidade de reação da política monetária.

Com o intuito de analisar a influência da inflação de diferentes grupos de bens e serviços foram efetuadas estimações que separam o IPCA em itens livres e administrados. A autoridade monetária deveria estar mais preocupada com as variações dos preços livres, uma vez que é o grupo de bens e serviços que são influenciados pela política monetária. Além disso, as variações da taxa de juros possuem pouca influência sobre os preços monitorados (OMOTO, DIAS e DIAS, 2008). As funções de reação das equações 4.4 e 4.5 são modificadas em relação à regra original, mas permitem analisar o grupo de bens e serviços que mais impactam na decisão de política monetária:

$$
\begin{aligned}
& i_{t}=\alpha_{1} i_{t-1}+\alpha_{2} i_{t-2}+\alpha_{3} i_{t-3}+\alpha_{4} y_{t}+\alpha_{5} E_{P a, j} \\
& i_{t}=\alpha_{1} i_{t-1}+\alpha_{2} i_{t-2}+\alpha_{3} i_{t-3}+\alpha_{4} y_{t}+\alpha_{5} E_{P l, j}
\end{aligned}
$$


Tabela 5: Estimativas da função de reação separada em preços administrados e preços livres Variável Dependente: $i_{t}$

Função de Reação com Preços Administrados
Função de Reação com Preços Livres

\begin{tabular}{ccccc} 
Variáveis & Coeficientes & Erro-Padrão & Coeficientes & Erro-Padrão \\
\cline { 2 - 5 }$i_{t-1}$ & $1,7042^{* * *}$ & $(0,1648)$ & $1,9325^{* * *}$ & $(0,1461)$ \\
$i_{t-2}$ & $-1,0569^{* * *}$ & $(0,2456)$ & $-1,2974^{* * *}$ & $(0,2429)$ \\
$i_{t-3}$ & $0,2555^{* *}$ & $(0,1148)$ & $0,3547^{* * *}$ & $(0,1144)$ \\
$y_{t}$ & $0,0696^{\mathrm{ns}}$ & $(0,0458)$ & $0,0752^{\mathrm{ns}}$ & $(0,0491)$ \\
$E_{P a, j}$ & $0,2489^{* *}$ & $(0,1100)$ & & \\
$E_{P l, j}$ & & & $0,0766^{\mathrm{ns}}$ & $(0,0886)$ \\
\hline $\mathrm{R}^{2}$ Ajustado & 0,9771 & \multicolumn{2}{c}{0,9742} \\
Observações & 39 & \multicolumn{3}{c}{39} \\
\hline
\end{tabular}

Testes de Especificação (p-valores)

$\begin{array}{ccc}\text { ARCH } & 0,6324 & 0,7242 \\ \text { LM } & 0,2709 & 0,3795 \\ \text { JB } & 0,6260 & 0,7894\end{array}$

Nota: ${ }^{*}$ Significativo a $10 \%,{ }^{* *}$ Significativo a $5 \%,{ }^{* * *}$ Significativo a $1 \%,{ }^{\text {ns }}$ Nãosignificativo. ARCH refere-se ao teste de presença de heterocedasticidade dos resíduos estimados. LM refere-se ao teste de presença de autocorrelação serial dos resíduos estimados. JB refere-se ao teste de normalmente dos resíduos estimados. AS estimativas utilizam séries temporais com dados trimestrais.

Fonte: Elaboração própria/Eviews.

As estimativas cujos resultados são mostrados na tabela 5 revelam que somente os preços administrados influenciam significativamente na decisão de política monetária que considera o período todo do regime de metas para inflação. Da mesma forma que a expectativa de inflação dos itens livres, o hiato do produto apresentou-se não significativo para influenciar na determinação da taxa de juros. Considerando a equação estimada dos preços administrados, um aumento de 1\% das expectativas ponderadas referentes os preços administrados apresenta impacto de 0,24 pontos percentuais na determinação da taxa de juros de curtíssimo prazo, revelando uma preferência assimétrica do Copom em relação aos preços administrados. 


\subsection{Estimativas dos Modelos Teóricos: Análises para os diferentes períodos presidenciais}

Por fim, é interessante analisar as preferências de política monetária das diferentes gestões do BCB através da estimação de uma função de reação para cada período presidencial. As equações dizem respeito às gestões de Armínio Fraga, Henrique Meirelles e Alexandre Tombini, respectivamente:

$$
\begin{aligned}
& i_{t}=\alpha_{1} i_{t-1}+\alpha_{2}\left(\pi_{t-1}-\pi_{t-1}^{*}\right)+\alpha_{3} E_{y, j}+\alpha_{4} D_{\pi, 2002} \\
& i_{t}=\alpha_{1} i_{t-1}+\alpha_{2} i_{t-2}+\alpha_{3} E_{\pi, j}+\alpha_{4} y_{t}+\alpha_{5} D_{y, 2003} \\
& i_{t}=\alpha_{1} i_{t-1}+\alpha_{2} E_{\pi, j}+\alpha_{3} y_{t}
\end{aligned}
$$

\begin{tabular}{|c|c|c|c|c|c|c|}
\hline & & ariável D & pendente: & & & \\
\hline & A. Fr & & H. Me & elles & A. Ton & bini \\
\hline Variáveis & Coef. & $\begin{array}{l}\text { Erro- } \\
\text { Padrão }\end{array}$ & Coef. & $\begin{array}{l}\text { Erro- } \\
\text { Padrão }\end{array}$ & Coef. & $\begin{array}{l}\text { Erro- } \\
\text { Padrão }\end{array}$ \\
\hline$i_{t-1}$ & $0,9561^{* * * *}$ & 0,0107 & $1,1877^{* * *}$ & 0,099 & $0,9148^{* * *}$ & 0,0201 \\
\hline$i_{t-2}$ & & & $-0,1944^{* *}$ & 0,0979 & & \\
\hline$E_{\pi, j}$ & & & $0,0547^{*}$ & 0,0349 & $0,7322^{* * *}$ & 0,1770 \\
\hline$\pi_{t-1}-\pi_{t-1}^{*}$ & $0,3375^{* * * *}$ & 0,0789 & & & & \\
\hline$y_{t}$ & & & $0,0648^{* * *}$ & 0,0164 & $0,0646^{*}$ & 0,0362 \\
\hline$E_{y, j}$ & $0,2679^{* * *}$ & 0,0845 & & & & \\
\hline$D_{\pi, 2002}$ & $0,0204^{* * *}$ & 0,003 & & & & \\
\hline$D_{y, 2003}$ & & & $-0,011^{* * *}$ & 0,0021 & & \\
\hline $\mathrm{R}^{2}$ Ajustado & 0,94 & & 0,99 & & 0,97 & \\
\hline Observações & 35 & & 9 & & 35 & \\
\hline
\end{tabular}

Tabela 6: Estimativas da função de reação dos diferentes governos presidenciais

Testes de Especificação (p-valores)

$\begin{array}{cccc}\text { ARCH } & 0,5699 & 0,1499 & 0,4579 \\ \text { LM } & 0,4792 & 0,2014 & 0,9571 \\ \text { JB } & 0,2927 & 0,5917 & 0,5077\end{array}$

Nota: ${ }^{*}$ Significativo a $10 \%,{ }^{* *}$ Significativo a $5 \%,{ }^{* * *}$ Significativo a $1 \%,{ }^{\text {ns }}$ Não-significativo. ARCH refere-se ao teste de presença de heterocedasticidade dos resíduos estimados. LM refere-se ao teste de presença de autocorrelação serial dos resíduos estimados. JB refere-se ao teste de normalmente dos resíduos estimados. As estimativas utilizam séries temporais com dados mensais.

Fonte: Elaboração própria/Eviews 
Conforme os resultados apresentados na tabela 6 , somente as gestões de Henrique Meirelles e Alexandre Tombini seguem uma função de reação com expectativas em relação à inflação futura. Pelos resultados na gestão de Armínio Fraga se utilizava mais das informações passadas dos desvios da inflação em relação à sua meta. Esta evidência pode ser explicada pela baixa confiabilidade das expectativas de mercado em relação à inflação futura nos primeiros anos do regime de metas, que em alguns momentos apresentaram-se distantes do dado observado. Entretanto, a gestão de Armínio Fraga também foi a única que se utilizou das variações futuras do hiato do produto em vez das observações passada.

O coeficiente $\alpha_{1}$ dos modelos estimados revela que em todos os casos o $\mathrm{BCB}$ agiu de forma a suavizar a política monetária ao longo do tempo sem provocar grandes choques na taxa nominal de juros entre dois períodos. Em relação às preferências distintas entre as gestões do $\mathrm{BCB}$, as estimativas mostram que Armínio Fraga e Alexandre Tombini agiram de modo mais prudente e preocupado com a estabilidade de preços. O período entre o último trimestre de 2002 e o terceiro trimestre 2003 mostram uma disparada do nível de preços da economia brasileira, principalmente dos preços administrados, o que pode ter contribuído para aumentar a preferência da autoridade monetária em relação à estabilidade de preços neste período. Por outro lado, a atuação de Henrique Meirelles revela uma maior preocupação com o emprego e produto, com o auxílio nos níveis menores de inflação. Chama a atenção o peso que a gestão de Alexandre Tombini atribui ao controle inflacionário, que cresce no ano de 2013 na medida em que a inflação persegue níveis próximos ao teto da meta (6,5\% a.a.). As dummies $D_{\pi, 2002} \mathrm{e}$ $D_{\pi, 2003}$ foram significativas em seus respectivos modelos, sendo que aquela influenciou positivamente o nível da taxa nominal de juros frente ao cenário de instabilidade política e intensa depreciação da moeda doméstica.

As estimações a seguir repetem a análise feita anteriormente em relação à taxa de inflação separada em preços livres e preços administrados, contudo diferenciado entre as gestões de Henrique Meirelles e Alexandre Tombini, respectivamente:

$$
\begin{aligned}
& i_{t}=\alpha_{1} i_{t-1}+\alpha_{2} i_{t-3}+\alpha_{3} i_{t-4}+\alpha_{4} E_{P a, j}+\alpha_{5} E_{P l, j}+\alpha_{6} E_{y, j}+\alpha_{7} D_{y, 2009} \\
& i_{t}=\alpha_{1} i_{t-1}+\alpha_{2} E_{P a, j}+\alpha_{3} E_{P l, j}+\alpha_{4} E_{y, j}+c
\end{aligned}
$$


A tabela 7 apresenta os resultados das estimativas. Ainda, foram estimados os coeficientes recursivos das expectativas em relação aos preços livres e administrados, procurando identificar períodos de mudança das preferências da política monetária, que são exibidos pelas figuras 3 e 4 .

Tabela 7: Estimativas da função de reação separada em preços administrados e livres dos diferentes governos presidenciais

\begin{tabular}{|c|c|c|c|c|}
\hline \multirow[b]{2}{*}{ Variáveis } & \multicolumn{2}{|c|}{$\begin{array}{l}\text { Variável Dependente: } i_{t} \\
\text { Lula }\end{array}$} & $i_{t}$ & Dilma \\
\hline & Coeficientes & $\begin{array}{c}\text { Erro- } \\
\text { Padrão }\end{array}$ & Coeficientes & $\begin{array}{c}\text { Erro- } \\
\text { Padrão }\end{array}$ \\
\hline$i_{t-1}$ & $1,0402^{* * *}$ & 0,0527 & $0,999^{* * *}$ & 0,0711 \\
\hline$i_{t-3}$ & $0,2907^{* *}$ & 0,1175 & & \\
\hline$i_{t-4}$ & $-0,3981^{* * *}$ & 0,0818 & & \\
\hline$E_{P a, j}$ & $0,1948^{* * *}$ & 0,0397 & $0,9275^{* *}$ & 0,4239 \\
\hline$E_{P l, j}$ & $0,1544^{* *}$ & 0,0714 & $1,3848^{* * *}$ & 0,3152 \\
\hline$E_{y, j}$ & $-0,0421^{\mathrm{ns}}$ & 0,0325 & $0,1178^{*}$ & 0,0651 \\
\hline$D_{y, 2009}$ & $-0,0049^{* * *}$ & 0,0011 & & \\
\hline $\mathrm{c}$ & & & $-0,0602^{* * *}$ & 0,0167 \\
\hline $\mathrm{R}^{2}$ Ajustado & 0,9 & & 0,9 & \\
\hline Observações & 8 & & 3 & \\
\hline \multicolumn{5}{|c|}{ Testes de Especificação (p-valores) } \\
\hline $\mathrm{ARCH}$ & \multicolumn{2}{|c|}{0,1826} & \multicolumn{2}{|c|}{0,6493} \\
\hline LM & \multicolumn{2}{|c|}{0,6500} & \multicolumn{2}{|c|}{0,2857} \\
\hline JB & \multicolumn{2}{|c|}{0,6704} & \multicolumn{2}{|c|}{0,5244} \\
\hline \multicolumn{5}{|c|}{$\begin{array}{l}\text { Nota: }{ }^{*} \text { Significativo a } 10 \%,{ }^{* *} \text { Significativo a } 5 \%,{ }^{* * *} \text { Significativo a } 1 \%,{ }^{\text {ns }} \text { Não- } \\
\text { significativo. ARCH refere-se ao teste de presença de heterocedasticidade dos resíduos } \\
\text { estimados. LM refere-se ao teste de presença de autocorrelação serial dos resíduos } \\
\text { estimados. JB refere-se ao teste de normalmente dos resíduos estimados. As estimativas } \\
\text { utilizam séries temporais com dados trimestrais. }\end{array}$} \\
\hline
\end{tabular}

Fonte: Elaboração própria/Eviews 
Figuras 3: Coeficientes recursivos da equação estimada 4.9
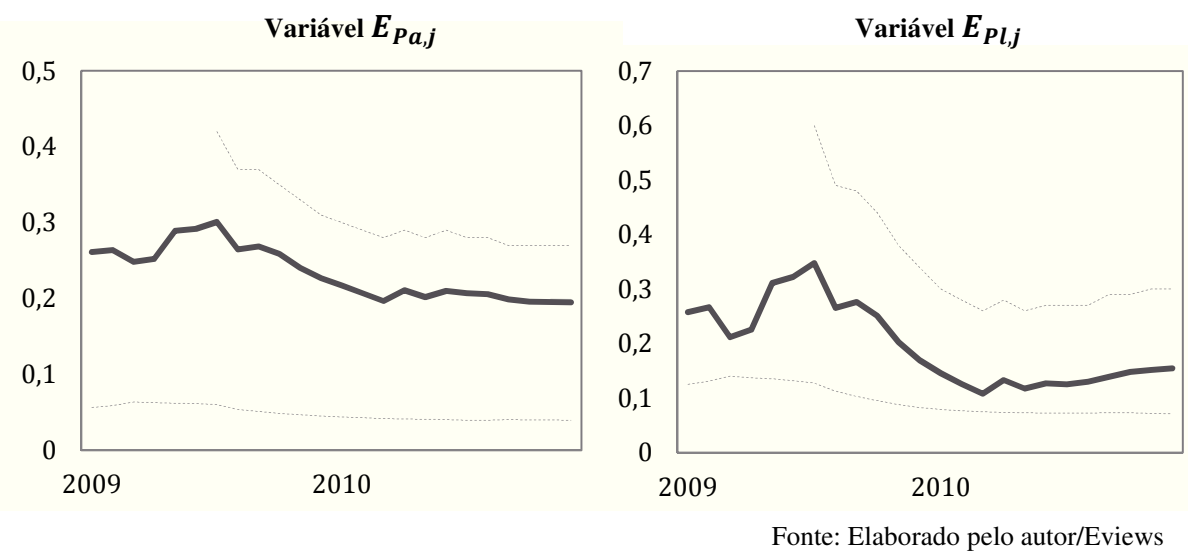

Figuras 4: Coeficientes recursivos da equação estimada 4.10
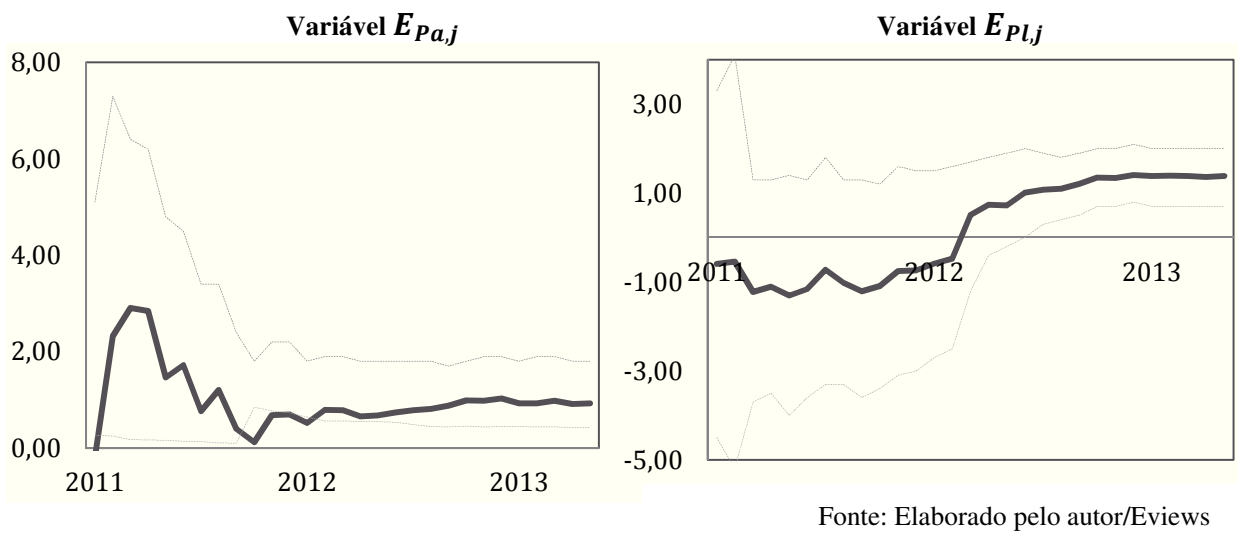

Os testes de especificação revelam resíduos estimados idênticos e independente distribuídos $\left(\varepsilon_{t} \sim\right.$ i.i.d $)$, tornando as estimativas das equações 4.9 e 4.10 válidas. Conforme as estimativas o Copom esteve mais preocupado com os movimentos dos preços administrados na gestão de Henrique Meirelles. Por outro lado, a gestão de Alexandre Tombini teve que enfrentar fortes pressões dos preços livres, principalmente de alimentos e bebidas e do setor de serviços ao longo de 2012 e 2013, o que pode explicar a preferência assimétrica ao controle desse grupo de preços. Essas conclusões são confirmadas pela figura 3 , indicando que os formuladores 
de política monetária na gestão Meirelles priorizaram o controle dos preços administrados nos primeiros anos de mandato. Os coeficientes recursivos também indicam que os preços em geral foram perdendo seu impacto sobre as decisões do Copom, revelando ganhos de potência da política monetária no decorrer do período. O movimento contrário é identificado no mandato de Tombini, figura 4, quando o movimento dos preços parece influenciar mais na determinação da taxa de juros de curto prazo, indicando perda de potência da política monetária nos últimos anos de governo.

A gestão de Alexandre Tombini exibe uma forte e instável preocupação com os preços administrados no início do período, enquanto que os preços livres ganham maior peso das decisões de política monetária a partir de 2012. O modelo estimado revela que um aumento de $1 \%$ nas expectativas dos preços livres geram um impacto significativo e positivo de 1,38 pontos percentuais na taxa Selic. Em geral, verifica-se uma forte correlação entre as preferências da função de reação do Banco Central com os valores absolutos da taxa de inflação, isto é, pressões fortes nos preços livres e administrados promovem mudanças significativas nos parâmetros de preferência da autoridade monetária.

\section{CONCLUSÕES}

O Banco Central do Brasil tem conduzido uma política monetária que prioriza a estabilização da inflação ao longo do regime de metas, mas que também atenta para os movimentos do hiato do produto e que suaviza intertemporalmente o instrumento de política. Em outras palavras, a autoridade monetária brasileira possui uma preferência assimétrica à estabilidade de preços, com maior prioridade aos preços administrados nas primeiras duas gestões do Banco Central e aos preços livres na gestão Tombini. Ainda, a taxa de juros Selic mostrou respostas lineares aos desvios esperados da inflação e produto às suas metas.

A estimativa da Curva de Phillips revela que tanto os movimentos do câmbio quanto do hiato do produto influenciam a inflação esperada. Isto significa que os agentes já incorporam os efeitos de pass-through da taxa de câmbio e do hiato do produto quando formam expectativas sobre a inflação futura. Por estar parcialmente incorporado nas expec- 
tativas da inflação futura, o hiato do produto não indicou impactos tão significativos nas decisões de política monetária quanto a própria inflação futura esperada.

Nos períodos de alta instabilidade financeira, com elevadas taxas de inflação tanto dos preços livres como administrados, o Banco Central do Brasil mostrou-se mais avesso às grandes flutuações da taxa de inflação. Ou seja, o policymaker atribuiu maior peso à estabilidade de preços quando acontecem choques significativos nas expectativas do nível de preços futuro da economia.

Com o passar do regime de metas, com exceção dos últimos anos da gestão Tombini, o valor absoluto dos coeficientes da função de reação reduziram, indicando queda do impacto da expectativa de inflação e do hiato do produto sobre a decisão de política monetária. Esse resultado acompanhado de análises qualitativas denota ganhos de potência da política monetária, motivado principalmente pelo fortalecimento dos canais de transmissão da política monetária.

\section{BEHAVIOR OF THE BRAZILIAN CENTRAL BANK REACTION FUNCTION: AN ANALYSIS FOR THE INFLATION TARGETING PERIOD}

\section{Abstract}

The reaction function of the Central bank expresses the preferences of the monetary authority with respect of deviations of his inflation target and the output gap. This paper evaluates the Brazilian Central Bank's preferences and its changes during the inflation targeting period. The model of Clarida, Galí and Gertler (1999) is employed in a forward looking version. Time series econometrics is the statistical tool. Results show that the preferences of the Brazilian Central Bank are asymmetrical with respect to its goals. The monetary policymaker is more sensitive to price stability, but also takes output in account and smooth's the interest rate over time. The monetary authority reacted stronger to controlled prices while Armínio Fraga and Henrique Meirelles were incumbent 
and to free prices during the Alexandre Tombini period. During financial instability periods the weight of price stability grows. There are signs that monetary policy power is increasing during the inflation targeting period, as also finds the Brazilian Central Bank.

Keywords: reaction function, preferences, monetary policy, Brazilian Central Bank, inflation targeting

J.E.L. Classification: E31, E32, E42, E52, E58

\section{REFERÊNCIAS}

ARAGON, E. K. S. B.; PORTUGAL, M. S. Central Bank preferences and monetary rules under the inflation targeting regime in Brazil. Brazilian Review of Econometrics, v. 29, n. 1, p. 79-109, 2009.

ARAGON, E. K. S. B.; PORTUGAL, M. S. Nonlinearities in Central Bank of Brazil's reaction function: the case of asymmetric preferences. Estudos Econômicos, v. 40, n. 2, 2010.

BUENO, R.L.S. Econometria de Séries Temporais. Cengage Learning, São Paulo, 2a edição revista e atualizada, 2012.

CLARIDA, R.; GALÍ, J; GERTLER, M. The science of monetary policy: a new Keynesian perspective. Cambridge: National Bureau of Economic Research, Working Paper 7147, 1999.

CLARIDA, R.; GALÍ, J; GERTLER, M. Monetary policy rules and macroeconomic stability: evidence and some theory. Quarterly Journal of Economics, v. 115, n. 1, 2000.

CUKIERMAN, A. The inflation bias result revisited. Tel-Aviv University, 2000. 
HENDRY, D. F. Achievements and challenges in econometric methodology, Journal of Econometrics, v. 100, pp. 7-10, 2001.

MEDEIROS, G. B.; ARAGÓN, E. K. S. B. Testando assimetrias nas preferências do Banco Central em uma pequena economia aberta: um estudo para o Brasil. Encontro Nacional de Economia, 34. Anais. Foz do Iguaçu, 2011.

MINELLA, A.; FREITAS, P. S.; GOLDFAJN, I.; MUINHOS, M. Inflation targeting in Brazil: constructing credibility under exchange rate volatility. Journal of International Money and Finance, v. 22, p. 1015-1040, 2003.

MINELLA, A.; FREITAS, P. S.; GOLDFAJN, I.; MUINHOS, M. Inflation targeting in Brazil: lessons and challenges, Central Bank of Brazil, Working Paper Series n. 53, Nov., 2002.

OMOTTO, K.H.; DIAS, M.H.A.; DIAS, J. Efeitos dos choques de política monetária sobre a atividade econômica e os preços no Brasil. XI Encontro Regional de Economia ANPEC-Sul, 2008.

TAYLOR, J.B. Discretion versus policy rules in practice. In CarnegieRochester Conference Series on Public Policy, v. 39, 1993.

TOSTES, F. S. O efeito da credibilidade sobre o pass-through da taxa de câmbio: uma análise para o Brasil sob metas para inflação. Tese de doutorado, Programa de Pós Graduação em Economia, Universidade Federal Fluminense, Niterói, 2014.

WOOLDRIDGE, J.M. Introdução à Econometria - Uma Abordagem Moderna. Cengage Learning, tradução da $4^{\text {a }}$ edição norte-americana, 2011. 